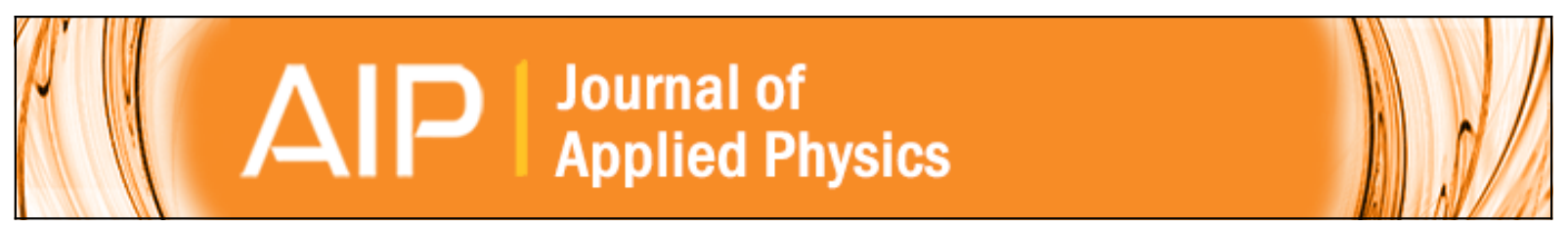

\title{
Shift of the blocking temperature of Co nanoparticles by Cr capping
}

M. Ewerlin, O. Petracic, D. Demirbas, L. Agudo, G. Eggeler, F. Brüssing, R. Abrudan, and H. Zabel

Citation: Journal of Applied Physics 114, 233908 (2013); doi: 10.1063/1.4851677

View online: http://dx.doi.org/10.1063/1.4851677

View Table of Contents: http://scitation.aip.org/content/aip/journal/jap/114/23?ver=pdfcov

Published by the AIP Publishing

\section{Articles you may be interested in}

Interaction effects and transport properties of Pt capped Co nanoparticles

J. Appl. Phys. 113, 043917 (2013); 10.1063/1.4789422

Correlation between saturation magnetization, bandgap, and lattice volume of transition metal ( $\mathrm{M}=\mathrm{Cr}, \mathrm{Mn}, \mathrm{Fe}$, Co, or Ni) doped Zn 1 - x M x O nanoparticles

J. Appl. Phys. 107, 09E314 (2010); 10.1063/1.3360189

Phase separation and nanoparticle formation in Cr-dosed FePt thin films

J. Appl. Phys. 101, 053901 (2007); 10.1063/1.2436926

Microstructural and magnetic properties of $\mathrm{ZnO}: \mathrm{TM}(\mathrm{TM}=\mathrm{Co}, \mathrm{Mn})$ diluted magnetic semiconducting nanoparticles

J. Appl. Phys. 100, 104315 (2006); 10.1063/1.2360387

The influence of shape and structure on the Curie temperature of Fe and Co nanoparticles

J. Appl. Phys. 99, 08G703 (2006); 10.1063/1.2167636

Frustrated by

old technology?

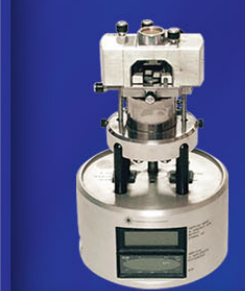

Is your AFM dead

and can't be repaired?

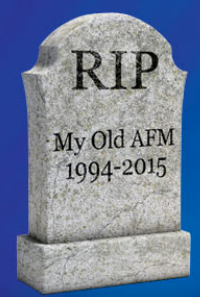

Sick of bad customer support?

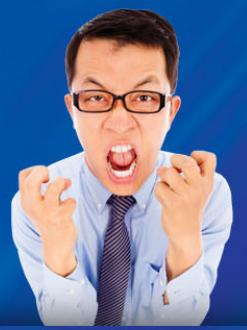

It is time to upgrade your AFM Minimum $\$ 20,000$ trade-in discount for purchases before August 31st

Asylum Research is today's technology leader in AFM

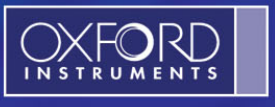




\title{
Shift of the blocking temperature of Co nanoparticles by $\mathrm{Cr}$ capping
}

\author{
M. Ewerlin, ${ }^{1}$ O. Petracic,,${ }^{1,2}$ D. Demirbas, ${ }^{1}$ L. Agudo, ${ }^{3}$ G. Eggeler, ${ }^{3}$ F. Brüssing, ${ }^{1}$ \\ R. Abrudan, ${ }^{1}$ and H. Zabel ${ }^{1}$ \\ ${ }_{1}^{1}$ Institute for Condensed Matter Physics, Ruhr-University Bochum, 44780 Bochum, Germany \\ ${ }^{2} J$ ülich Centre for Neutron Science JCNS-2 and Peter Grüberg Institute PGI-4, \\ Forschungszentrum Jülich GmbH, 52425 Jülich, Germany \\ ${ }^{3}$ Institute for Material Science, Ruhr-University Bochum, 44780 Bochum, Germany
}

(Received 22 November 2013; accepted 4 December 2013; published online 19 December 2013)

\begin{abstract}
We have studied the effect of Cr capping on the magnetic properties of Co nanoparticles (NPs). The NPs have an average diameter of $2.2 \mathrm{~nm}$. The blocking temperature $\mathrm{T}_{B}$ of the bare Co particles is $13.2 \mathrm{~K}$. By capping with a thin Cr layer up to a thickness of $\mathrm{t}_{C r}=0.52 \mathrm{~nm}$, we first observe a decrease of $\mathrm{T}_{B}$ up to $\mathrm{t}_{C r}=0.14 \mathrm{~nm}$, followed by an increase of $\mathrm{T}_{B}$ for larger thicknesses $0.14 \mathrm{~nm} \leq$ $\mathrm{t}_{C r} \leq 0.52 \mathrm{~nm}$. X-ray magnetic circular dichroism measurements at the resonant Co and $\mathrm{Cr} L_{3}$ edges confirm a magnetic polarization of $\mathrm{Cr}$ which is opposite to the magnetization of $\mathrm{Co}$. The antiparallel alignment of $\mathrm{Co}$ and $\mathrm{Cr}$ spins at the $\mathrm{Co} / \mathrm{Cr}$ interface can explain the decrease at low capping layer thickness. However, for larger $\mathrm{Cr}$ capping layer thicknesses, the $\mathrm{Cr}$ film bridges the Co NPs, mediating interparticle exchange coupling and enhancing dipolar coupling that leads to an increase of the blocking temperature. (C) 2013 AIP Publishing LLC. [http://dx.doi.org/10.1063/1.4851677]
\end{abstract}

\section{INTRODUCTION}

Magnetic nanoparticles (NPs) play an increasingly important role in many fields of nanomagnetism. ${ }^{1,2}$ In several cases, they are composed of a core and a shell, where the core may consist of a ferromagnetic metal covered by its natural oxide, a ferri- or antiferromagnetic oxide surrounded by a diluted antiferromagnet, ${ }^{3}$ or a capping layer for oxidation protection. In any case, the properties of magnetic NPs strongly depend on the interaction between core and shell or the surrounding. Magnetic NPs may either be produced in the gas phase and mass selected subsequentially, they may be embedded into a fluid or polymer matrix, or they may be deposited on a substrate. Deposition on a well chosen substrate has several advantages as the growth mode and self-assembly of the NPs can be tuned by the interface energy between NPs and substrate. ${ }^{4}$ In the literature, it was shown that Co deposited on sapphire buffer layers form small non-overlapping clusters with a rather narrow size distribution for effective film thicknesses below $2 \mathrm{~nm} .{ }^{5-10}$ When capping these NPs with a thin Pt layer, the blocking temperature is dramatically increased from below $10 \mathrm{~K}$ up to room temperature. ${ }^{9,10}$ In the following, we describe the magnetic properties of similar Co NPs, but covered with different $\mathrm{Cr}$ film thicknesses. Cr plays a prominent role as an exchange coupling medium in $\mathrm{Fe} / \mathrm{Cr}^{11-13}$ and $\mathrm{Co} / \mathrm{Cr}^{14-16}$ multilayers. It has also been used in giant magnetoresistive spin valve devices and for increasing the coercivity in hard disks. ${ }^{17}$ Furthermore, Cr is often used as a capping layer for oxidation protection. ${ }^{18}$ It is therefore of interest to scrutinize the interaction of Cr capping layers with Co nanoclusters and to decide whether the blocking temperature is enhanced or reduced with $\mathrm{Cr}$ capping.

\section{EXPERIMENTAL METHODS AND RESULTS}

\section{A. Sample preparation}

The Co NPs were prepared by sequential ion beam sputter deposition of aluminum oxide $\left(\mathrm{Al}_{2} \mathrm{O}_{3}\right)$ and $\mathrm{Co}$ with highly purified Ar gas. All samples were prepared at a base pressure better than $5 \times 10^{-9}$ mbar at room temperature. $\mathrm{Si}$ covered with its natural oxide was used as substrate. Co NPs are formed by aggregation due to an extreme Volmer-Weber growth mode of the metal atoms sputter deposited on amorphous sapphire. ${ }^{5-7}$ After sputtering, a $5 \mathrm{~nm}$ amorphous $\mathrm{Al}_{2} \mathrm{O}_{3}$ layer on $\mathrm{Si}$, Co was sputtered on top with a nominal thickness of $\mathrm{t}_{C o}=0.52 \mathrm{~nm}$. Both, $\mathrm{Al}_{2} \mathrm{O}_{3}$ and $\mathrm{Co}$ were sputtered at an angle close to perpendicular incidence. The particles were capped by a $\mathrm{Cr}$ layer of varying nominal thickness between $\mathrm{t}_{C r}=0 \mathrm{~nm}$ and $\mathrm{t}_{C r}=0.52 \mathrm{~nm}$. The $\mathrm{Cr}$ film was deposited at an angle of $62^{\circ}$ with respect to the sample normal. This procedure is schematically illustrated in Fig. 1. Due to this steep angle, the Co atoms serve as shadow masks for the arriving chromium atoms. This leads for very thin Cr films to a capping of only the Co particles, whereas with increasing $\mathrm{Cr}$ film thicknesses also the space in between the Co particles will be filled. Finally, all samples were capped with $5 \mathrm{~nm} \mathrm{Al}_{2} \mathrm{O}_{3}$ deposited at normal incidence, in order to embed the particles and prevent them from oxidation.

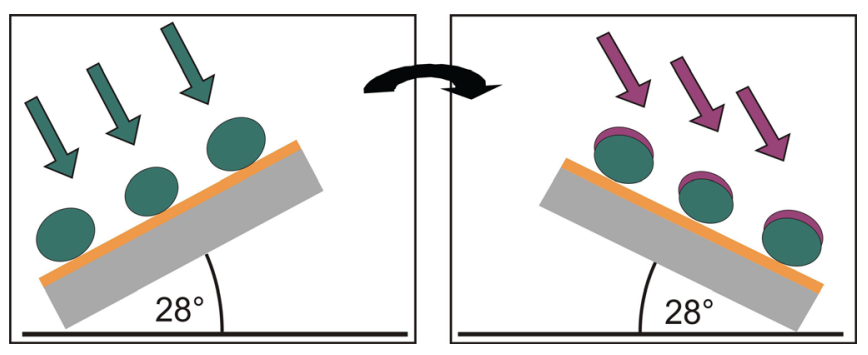

FIG. 1. Schematic illustration of the sample preparation. The substrate is inclined by $28^{\circ}$. The left hand panel shows the deposition of the Co nanoparticles at perpendicular incidence. Rotation of the sample by $180^{\circ}$ provides an incident angle of $62^{\circ}$ for the $\mathrm{Cr}$ deposition, as shown in the right hand panel. 

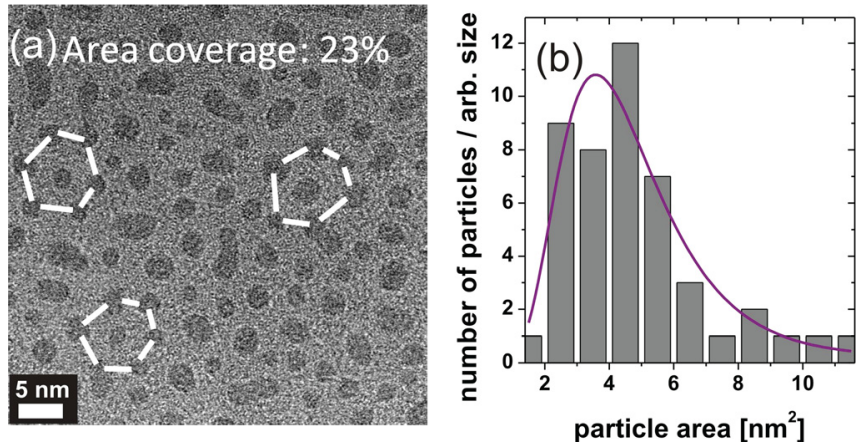

FIG. 2. (a) TEM image of uncapped Co particles. Scale bar is shown at the bottom. The white bars in the image indicate the roughly hexagonal order of the Co nanoparticles; (b) histogram of Co particle area. Solid curve is a fit to the distribution by a log normal plot.

\section{B. Structural and magnetic characterization}

The structural characterization of the samples was performed by means of transition electron microscopy (TEM) and scanning TEM (STEM) using an Analytical FEG-TEM TECNAI F20 S-Twin instrument working at $200 \mathrm{kV}$. For the TEM studies, additional samples were prepared using $\mathrm{KBr}$ instead of $\mathrm{Si}$ as substrate. The different substrates do not affect the growth properties of the nanoparticles, since the Co NPs are still deposited on the $\mathrm{Al}_{2} \mathrm{O}_{3}$ buffer layer. After deposition, the $\mathrm{KBr}$ substrate was dissolved in purified water and the film fragments were placed on Cu TEM grids. The magnetic characterization of the uncapped and capped particles was performed using a superconducting quantum interference device (SQUID) magnetometer (MPMS, Quantum Design) and x-ray resonant magnetic scattering (XRMS), performed at the synchrotron BESSY II of the Helmholtz-Zentrum Berlin (HZB) using the Alice chamber. ${ }^{19}$

\section{RESULTS}

\section{A. Particle distribution}

Fig. 2(a) shows a TEM image of the uncapped Co NPs with a nominal thickness of $t_{C o}=0.52 \mathrm{~nm}$. The particles show essentially a circular shape and are evenly distributed over the substrate. The overall coverage is about $23 \%$, and a quasi hexagonal order can be identified as expected., ${ }^{5,6}$ The size distribution of the particles was determined from the TEM images and a histogram showing the number of particles versus the particles area is plotted in Fig. 2(b). The data are fitted using a log normal plot (purple line). The size distribution allows to determine the mean diameter of the cobalt nanoparticles under the assumption of circular shape to be $d_{\text {mean }}=2.2 \mathrm{~nm}$. These results are in good agreement with literature values. ${ }^{5,6} \mathrm{Fig} .3$ shows the morphology of several samples with different amounts of Co NP capping by $\mathrm{Cr}$, i.e., (a) $\mathrm{t}_{C r}=0 \mathrm{~nm}$, (b) $\mathrm{t}_{C r}=0.14 \mathrm{~nm}$, (c) $\mathrm{t}_{C r}=0.35 \mathrm{~nm}$, and (d) $\mathrm{t}_{C r}=0.52 \mathrm{~nm}$. The upper two panels in each column show TEM images with different magnification, whereas the bottom panels show STEM images with enhanced contrast.

\section{B. Blocking temperature}

The effects of $\mathrm{Cr}$ capping on the magnetism of the Co particles were investigated by means of SQUID magnetometry. Several series of samples were prepared and the blocking temperature was estimated from zero field cooling (ZFC) and field cooling (FC) magnetization measurements in a field of 20 Oe. ${ }^{1}$ In Fig. 4, we show the results from uncapped Co particles with different Co thicknesses. The blocking temperatures-defined to be the temperature where the ZFC curve shows its maximum-range from $13.5 \mathrm{~K}$ for the smallest Co particles of $\mathrm{t}_{C o}=0.52 \mathrm{~nm}$ effective thickness to above room temperature for $\mathrm{t}_{C o}=1.56 \mathrm{~nm}$. These results, showing a
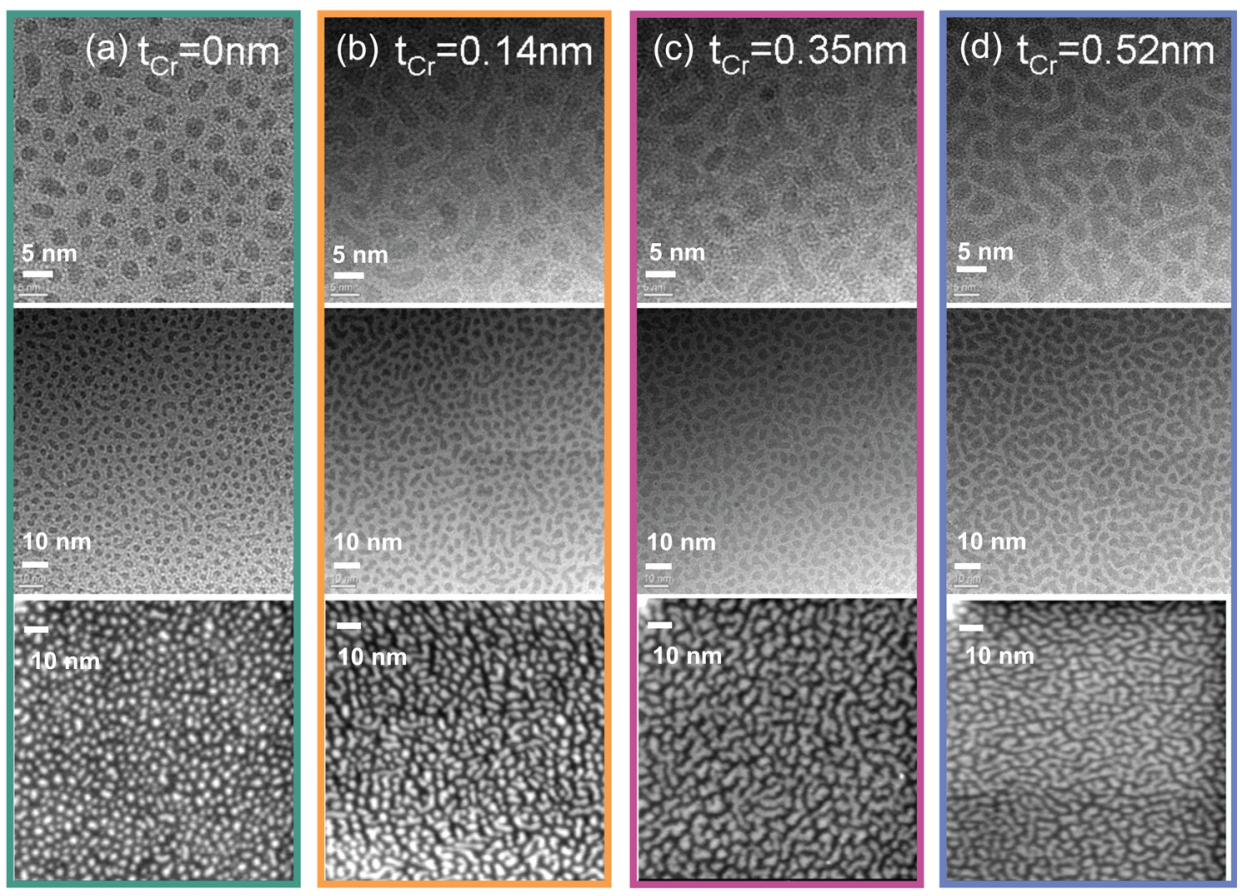

FIG. 3. TEM images (top and middle panels) and STEM (bottom panels) of Co nanoparticles with $\mathrm{t}_{C o}=0.52 \mathrm{~nm}$. Column (a): uncapped Co NPs; column (b): Co NPs capped with $\mathrm{t}_{C r}=0.14 \mathrm{~nm}$; column (c): Co NPs capped with $\mathrm{t}_{C r}=0.35 \mathrm{~nm} ;$ column (d): Co NPs capped with $\mathrm{t}_{C r}=0.52 \mathrm{~nm}$. The colored border around each column corresponds to the same color coding of the magnetization measurements plotted in Fig. 5 and the blocking temperatures plotted in Fig. 6. 

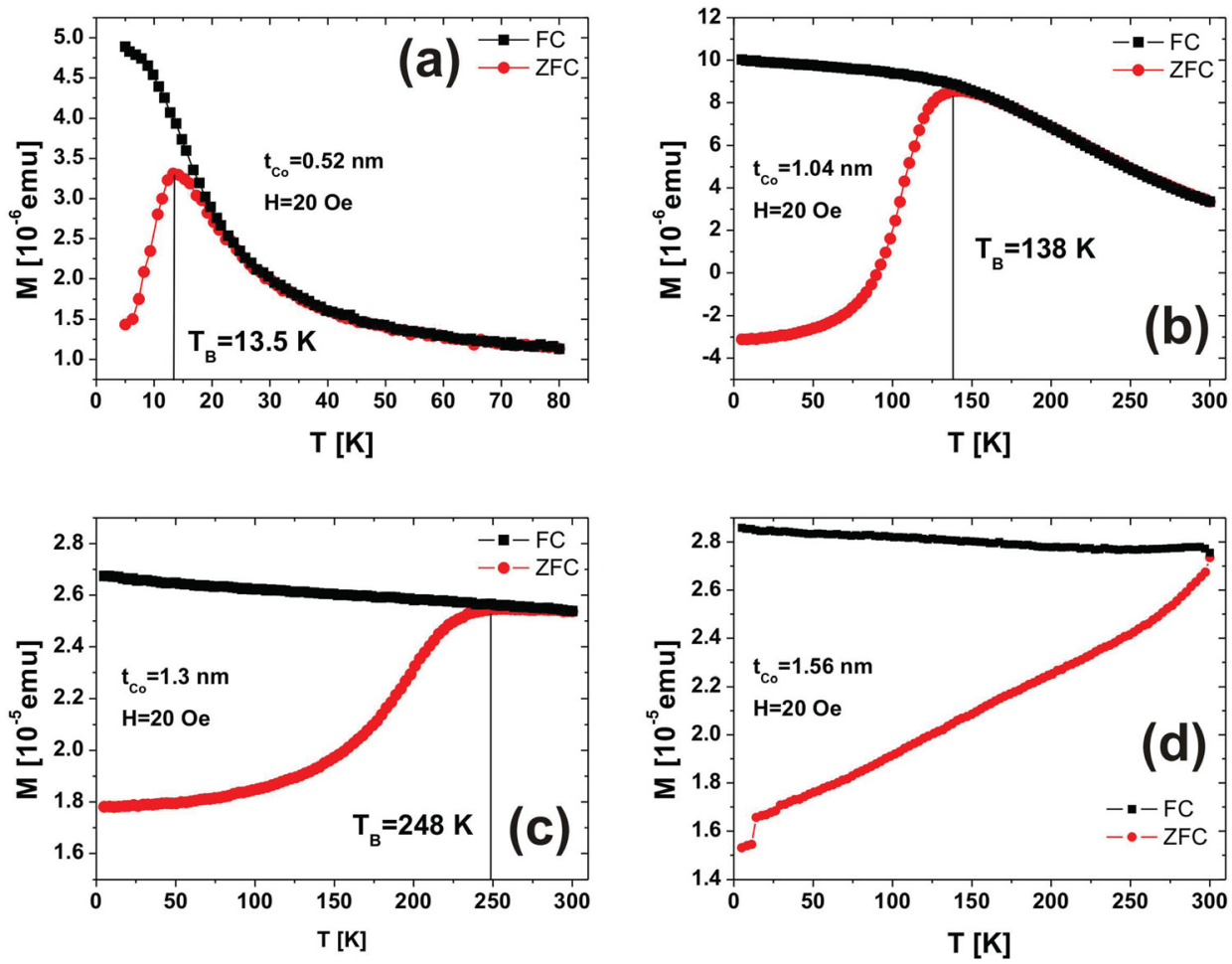

FIG. 4. M vs. T curves after ZFC (red circles) and FC (black squares) for various Co nanoparticles: (a) $\mathrm{t}_{C o}=0.52 \mathrm{~nm}$; (b) $\mathrm{t}_{C o}=1.04 \mathrm{~nm}$; (c) $\mathrm{t}_{C o}=1.3 \mathrm{~nm}$; (d) $\mathrm{t}_{C o}=1.56 \mathrm{~nm}$.

dramatic size dependence of the blocking temperature, confirm similar experiments by Ludwig and coworkers. ${ }^{10}$

Next, we concentrate on the thinnest Co nanoclusters with $\mathrm{t}_{C o}=0.52 \mathrm{~nm}$. Fig. 5 show $\mathrm{M}$ versus $\mathrm{T}$ curves taken again after $\mathrm{FC}$ and $\mathrm{ZFC}$ for uncapped particles and particles capped with (a) $\mathrm{t}_{C r}=0.0 \mathrm{~nm}, \quad$ (b) $\mathrm{t}_{C r}=0.14 \mathrm{~nm}, \quad$ (c) $\mathrm{t}_{C r}=0.35 \mathrm{~nm}$, and (d) $\mathrm{t}_{C r}=0.52 \mathrm{~nm}$, respectively. All samples show superparamagnetic behavior independent of the $\mathrm{Cr}$ capping thickness. This is different from capping Co NPs with Pt films, where for Pt thicknesses larger than $1.4 \mathrm{~nm}$ a transition from superparamagnetic to ferromagnetic behavior is observed. ${ }^{10}$ In the present case, however, the blocking temperature $\mathrm{T}_{B}$ first drops from the uncapped value $\mathrm{T}_{B}=13.2 \mathrm{~K}$ (Fig. $5(\mathrm{a})$ ) to $\mathrm{T}_{B}=11.2 \mathrm{~K}$ for a capping thickness of $\mathrm{t}_{C r}=0.14 \mathrm{~nm}$ (Fig. 5(b)). Increasing the capping thickness, this trend is reversed and an increase of $\mathrm{T}_{B}$ is observed: $\mathrm{T}_{B}=14.2 \mathrm{~K}$ for $\mathrm{t}_{C r}=0.35 \mathrm{~nm}$ (Fig. 5(c)) and $\mathrm{T}_{B}=19.2 \mathrm{~K}$ for $\mathrm{t}_{C r}=0.52 \mathrm{~nm}$ (Fig. $5(\mathrm{~d})$ ).

The blocking temperatures determined for all series of samples are plotted in Fig. 6 as a function of the nominal $\mathrm{Cr}$
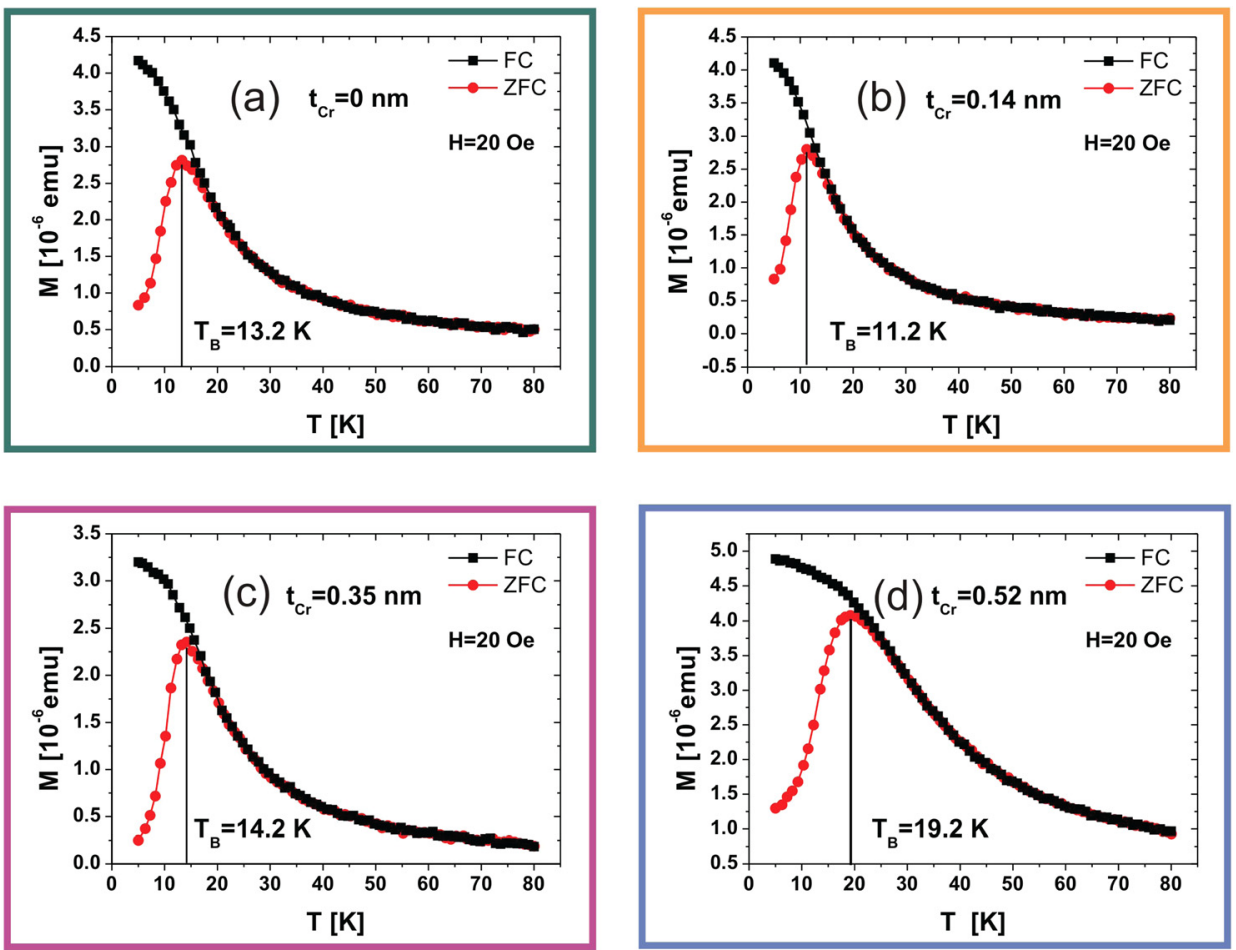

FIG. 5. M vs. T curves after ZFC and FC of Co nanoparticles with thickness $\mathrm{t}_{C o}=0.52 \mathrm{~nm}$ and for various amounts of $\mathrm{Cr}$ capping: (a) $\mathrm{t}_{C r}=0 \mathrm{~nm}$; (b) $\mathrm{t}_{C r}=0.14 \mathrm{~nm} ; \quad$ (c) $\mathrm{t}_{C r}=0.36 \mathrm{~nm} ; \quad$ (d) $\mathrm{t}_{C r}=0.52 \mathrm{~nm}$. 


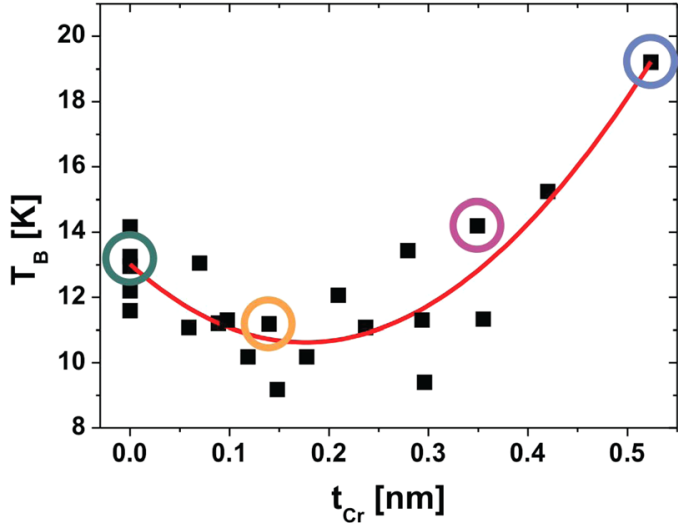

FIG. 6. Blocking temperature of Co nanoparticles versus thickness of $\mathrm{Cr}$ capping layer thickness. The encircled points correspond to the respective measurements shown in Figs. 3 and 5.

thickness. The encircled data points belong to the samples shown in Figs. 4 and 5 for reasons of recognition, whereas the red line is a fit through the data points and serves as a guide to the eye. Depending on the amount of Cr capping, the behavior can clearly be divided into two regimes: for small $\mathrm{t}_{C r}$ up to $\mathrm{t}_{C r}=0.14 \mathrm{~nm}$, a decrease of $\mathrm{T}_{B}$ is observed, whereas for higher amounts the capping leads to an enhancement of $\mathrm{T}_{B}$.

We concentrate on the thin $\mathrm{Cr}$ thickness regime first. In case of tungsten a reduction of the blocking temperature of Co NPs has also been observed, ${ }^{20,21}$ which is due to alloying with Co. However, $\mathrm{Cr}$ does not show an affinity to alloying with Co. ${ }^{22}$ Thus, another mechanism must be responsible for the reduction. It is well known that $\mathrm{Cr}$ at flat interfaces couples antiparallel to $\mathrm{Fe}$ and $\mathrm{Co}$ moments. ${ }^{23,24}$ It has to be shown whether this is also the case for $\mathrm{Cr}$ covering Co NPs and whether this can explain the reduction of the blocking temperature. Therefore, we have carried out $\mathrm{x}$-ray resonant magnetic scattering experiments at the $\mathrm{Co}$ and $\mathrm{Cr} L_{3}$ and $L_{2}$ resonance edges, respectively, as detailed in the Sec. III C. For larger $\mathrm{Cr}$ thicknesses, we suppose that $\mathrm{Cr}$ starts filling in the gaps between the NPs, providing an effective coupling medium between the NPs that will increase the blocking temperature. We will come back to this point in the Discussion (Sec. IV).

\section{X-ray magnetic circular dichroism}

The polarization of $\mathrm{Cr}$ with respect to $\mathrm{Co}$ was confirmed by means of x-ray resonant experiments at the Co and $\mathrm{Cr} L_{3}$ and $L_{2}$ resonant absorption edges. Since the amount of $\mathrm{Cr}$ is extremely small, $\mathrm{x}$-ray magnetic dichroism measurements (XMCD) in total electron yield detection mode (TEY) turned out not to be sufficiently sensitive, similar to our earlier experience for determining the $\mathrm{Cr}$ polarization in $\mathrm{Cr} / \mathrm{Co} / \mathrm{Cr}$ trilayer heterostructures. ${ }^{24}$ Therefore, we have chosen to perform the measurements in reflection geometry and to use the $\mathrm{x}$-ray resonant magnetic scattering configuration at small incident angles of $\theta=4.6^{\circ}$ and $4.96^{\circ}$ for $\mathrm{Co}$ and $\mathrm{Cr}$, respectively. These were the angles of maximum contrast between left and right circular polarized light or between positive and negative applied fields. The measurements were performed at a sample with $\mathrm{t}_{C r}=0.35 \mathrm{~nm}$. Although this thickness is already in the ascending branch of the blocking temperatures, it is still valid since the polarization at the interface is independent of the total amount of $\mathrm{Cr}$. The sample was cooled down to $40 \mathrm{~K}$ in an applied field of $2700 \mathrm{Oe}$, and energy scans were taken in positive and negative fields around the resonant Co edges (Co $L_{3}: 0.788 \mathrm{keV}$; $\left.\mathrm{Co} L_{2}: 0.792 \mathrm{keV}\right)$ and the resonant $\mathrm{Cr}$ edges $\left(\mathrm{Cr} L_{3}: 0.574 \mathrm{keV} ; L_{2}: 0.584 \mathrm{keV}\right)$, respectively. The respective energy scans are plotted in Figs. 7 and 8.

The upper part of the graphs shows the energy scans for $\mathrm{Co}$ and $\mathrm{Cr}$ in positive and negative fields, respectively, whereas the lower part shows the calculated asymmetry. Both the energy scans and the asymmetry resemble respective XMCD scans in absorption geometry. ${ }^{25,26}$ This is probably due to the very thin film thickness such that interference effects at small angles are negligible. The asymmetry for Co is very well pronounced. A clear $\mathrm{Cr}$ asymmetry is visible at the $\mathrm{Cr} L_{3}$ edge of $0.574 \mathrm{keV}$. This signal shows an opposite sign as compared to the Co signal. Although the signal is very low and noisy due to the small amount of material, a polarization of $\mathrm{Cr}$ can be confirmed and gives evidence for an antiparallel alignment of $\mathrm{Cr}$ magnetic moments with respect to Co moments at the interface. This is in complete agreement with a similar recent study by Brüssing and coworkers on $\mathrm{Cr} / \mathrm{Co} / \mathrm{Cr}$ heterostructures with extended $\mathrm{Co} / \mathrm{Cr}$ interfaces. ${ }^{24} \mathrm{We}$ attribute the decrease of the blocking temperature to this antiparallel alignment of the Co and $\mathrm{Cr}$ magnetic moments.

\section{DISCUSSION AND CONCLUSION}

In this work, Co nanoparticles with an effective thickness of $0.52 \mathrm{~nm}$ were covered with $\mathrm{Cr}$ capping layers of increasing thickness. The aim was to determine the change of the Co blocking temperature as function of the $\mathrm{Cr}$ capping layer thickness. Unlike most other capping layers, the blocking temperature immediately decreases when covered with

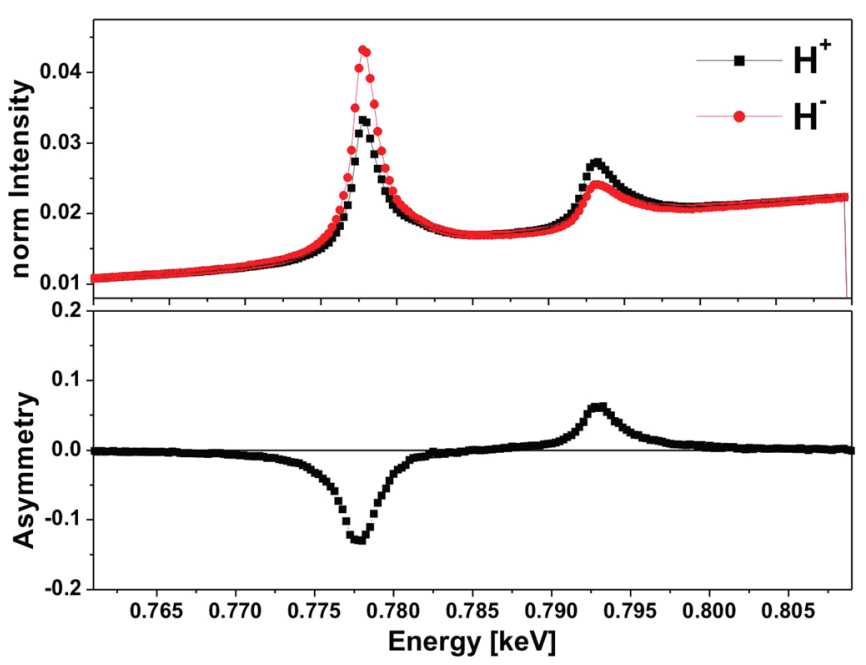

FIG. 7. Top panel: energy scan in reflection geometry near the Co $L_{3}$ and $L_{2}$ edges taken in positive and negative magnetic fields of $2700 \mathrm{Oe}$ at a temperature of $40 \mathrm{~K}$. The intensity is normalized to the monitor counts. Bottom panel: asymmetry signal of Co derived from the reflectivity data. 


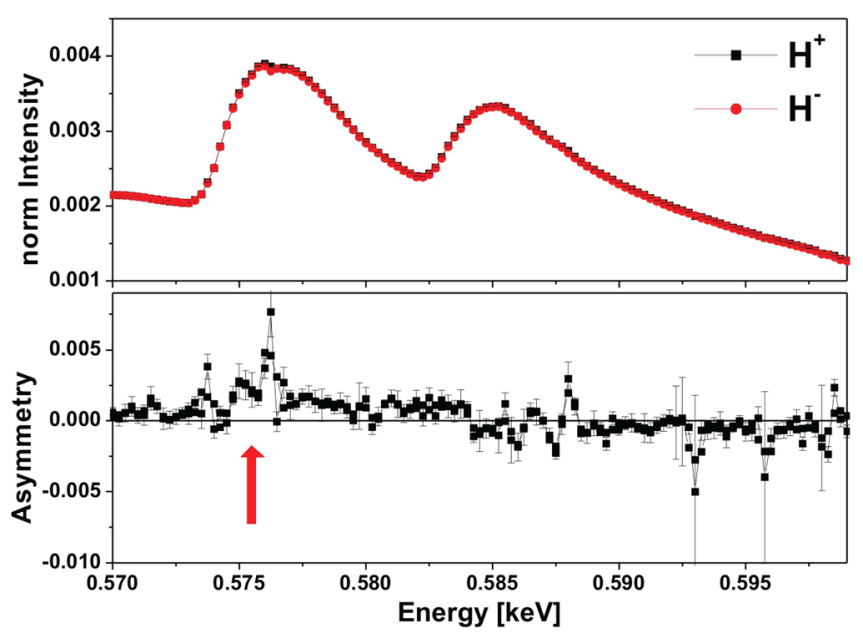

FIG. 8. Top panel: energy scan in reflection geometry near the $\mathrm{Cr} L_{3}$ and $L_{2}$ edges taken in positive and negative magnetic saturation field of the Co nanoparticles at $40 \mathrm{~K}$. The intensity is normalized to the monitor counts. Bottom panel: asymmetry signal of $\mathrm{Cr}$ derived from the reflectivity data. The arrow indicates the energy region where the asymmetry is clearly positive and opposite to the asymmetry of Co.

$\mathrm{Cr}$ and only increases slightly at capping layer thickness of $0.2 \mathrm{~nm}$ and higher. As in the first regime, the nominal $\mathrm{Cr}$ thickness is extremely low, the reduction of the blocking temperature can be explained as follows: $\mathrm{Cr}$ is polarized by Co and aligned antiparallel with respect to the magnetization of the NP as confirmed by our XRMS results. This leads to a "compensation" of the magnetic moments at the interface between $\mathrm{Co}$ and $\mathrm{Cr}$ and due to this to a decrease of the effective magnetic volume of each particle. The energy barrier that the particles need to overcome in order to switch is proportional to the anisotropy constant and the magnetic volume, i.e., $\Delta \mathrm{E} \sim \mathrm{KV}$. Since the blocking temperature is proportional to the energy barrier, a decrease of the effective volume leads to a decrease of the energy barrier and thus to a decrease of the blocking temperature, as observed.

For higher $\mathrm{Cr}$ capping thicknesses, i.e., $\mathrm{t}_{C r}>0.14 \mathrm{~nm}$, the blocking temperature increases again. Since the polarization also takes place in this regime, an additional mechanism overcompensating the polarization effect needs to be invoked to explain this increase. The TEM images shown in Fig. 3 indicate the formation of $\mathrm{Cr}$ bridges between the $\mathrm{Co}$ NPs with increasing capping layer thickness. This may lead to a coupling of the NPs, although the nature of the coupling is not well understood yet and needs further investigation in future experiments. Luis and coworkers have shown that in case of $\mathrm{Cu}$ and $\mathrm{Au}$ capping the polarization of the noble metals by the Co particles can mediate a Ruderman-KittelKasuya-Yoshida (RKKY) interaction between NPs. ${ }^{8}$ This may also be the case for $\mathrm{Cr}$. The average distance between the Co NPs is about $2 \mathrm{~nm}$. The period of the oscillatory exchange coupling deduced from $\mathrm{Fe} / \mathrm{Cr}$ multilayers is $1.7 \mathrm{~nm} .{ }^{27}$ Because of the distribution of the NPs distances, a unique ferro- or antiferromagnetic coupling among the NPs mediated by the $\mathrm{Cr}$ capping is not obvious. Apart from RKKY interactions, also magnetic dipolar interaction is of immense importance. It was shown, that magnetic dipole interaction dominates RKKY interaction for Co particles with a diameter of about $2 \mathrm{~nm} .^{28,29}$ In the case of uncapped NPs, the dipolar fields exhibited due to the single domain state of the NPs are randomly distributed over the sample, since the magnetization orientation is random. However, as the paramagnetic susceptibility of $\mathrm{Cr}$ is positive, ${ }^{30}$ the dipolar field lines are enhanced in the $\mathrm{Cr}$ film, which, in turn, reinforces the dipolar interaction among the nanoparticles. Hence, the coupling leads to an increase of the effective magnetic volume and an increase of the blocking temperature as observed.

In conclusion, we have investigated the dependence of the blocking temperature of Co nanoparticles on the thickness of capping $\mathrm{Cr}$ films. Surprisingly, we found that the blocking temperature first drops from $13.4 \mathrm{~K}$ to $11 \mathrm{~K}$ from zero capping to a $\mathrm{Cr}$ thickness of $0.14 \mathrm{~nm}$. Beyond this thickness, the blocking temperature increases again and reaches values of $19 \mathrm{~K}$ for a $\mathrm{Cr}$ capping thickness of $0.5 \mathrm{~nm}$. The dropping blocking temperature is assigned to a $\mathrm{Cr}$ induced magnetic polarization that is opposite to the Co magnetization, reducing effectively the total magnetic volume of the nanoparticles. The reason for the subsequent increase of the blocking temperature is mainly due to RKKY and dipolar interaction between the Co nanoparticles mediated by $\mathrm{Cr}$ bridges between the particles.

\section{ACKNOWLEDGMENTS}

The authors are grateful to Peter Stauche for technical support during the sample preparation. We would like to thank the Deutsche Forschungsgemeinschaft for financial support of this work within the Sonderforschungsbereich 491 "Magnetic heterostructures: spin structures and spin transport." The operation of the ALICE reflectometer is supported by the BMBF Verbundforschung (05K10PC2), which is gratefully acknowledged. We are also thankful to the $\mathrm{HZB}$ Berlin for travel support under No. BMBF 05ES3XBA/5.

${ }^{1}$ O. Petracic, Superlattices Microstruct. 47, 569 (2010).

${ }^{2}$ O. Hellwig, L. J. Heyderman, O. Petracic, and H. Zabel, Springer Tracts Mod. Phys. 246, 189-234 (2013).

${ }^{3}$ M. J. Benitez, O. Petracic, H. Tysz, F. Schth, and H. Zabel, Phys. Rev. B 83, 134424 (2011).

${ }^{4}$ D. Mishra, M. J. Benitez, O. Petracic, G. A. B. Confalonieri, P. Szary, F. Brssing, K. Theis-Brhl, and H. Zabel, Nanotechnology 23, 055707 (2012).

${ }^{5}$ J.-L. Maurice, J. Briático, J. Carrey, F. Petroff, L. F. Schelp, and A. Vaurès, Philos. Mag. A 79, 2921 (1999).

${ }^{6}$ J. Briático, J.-L. Maurice, J. Carrey, D. Imhoff, F. Petroff, and A. Vaurès, Eur. Phys. J. D 9, 517 (1999).

${ }^{7}$ J. Bartolomé, L. M. García, F. Bartolomé, F. Luis, R. López-Ruiz, F. Petroff, C. Deranlot, F. Wilhelm, A. Rogalev, P. Bencok, N. B. Brookes, L. Ruiz, and J. M. González-Calbet, Phys. Rev. B 77, 184420 (2008).

${ }^{8}$ F. Luis, F. Petroff, J. M. Torres, L. M. García, J. Bartolomé, J. Carrey, and A. Vaurès, Phys. Rev. Lett. 88, 217205 (2002).

${ }^{9}$ A. Ebbing, O. Hellwig, L. Agudo, G. Eggeler, and O. Petracic, Phys. Rev. B 84, 012405 (2011).

${ }^{10}$ A. Ludwig, L. Agudo, G. Eggeler, A. Ludwig, A. D. Wieck, and O. Petracic, J. Appl. Phys. 113, 043917 (2013).

${ }^{11}$ G. Binasch, P. Grünberg, F. Saurenbach, and W. Zinn, Phys. Rev. B 39, 4828 (1989).

${ }^{12}$ M. N. Baibich, J. M. Broto, A. Fert, F. Nguyen Van Dau, F. Petroff, P. Etienne, G. Creuzet, A. Friederich, and J. Chazelas, Phys. Rev. Lett. 61, 2472 (1988).

${ }^{13}$ A. Schreyer, J. F. Ankner, Th. Zeidler, H. Zabel, M. Schäfer, J. A. Wolf, P. Grünberg, and C. F. Majkrzak, Phys. Rev. B 52, 16066 (1995). 
${ }^{14}$ N. Metoki, W. Donner, and H. Zabel, Phys. Rev. B 49, 17351 (1994).

${ }^{15}$ W. Donner, T. Zeidler, F. Schreiber, N. Metoki, and H. Zabel, J. Appl. Phys. 75, 6421 (1994).

${ }^{16}$ T. Zeidler, F. Schreiber, H. Zabel, W. Donner, and N. Metoki, Phys. Rev. B 53, 3256 (1996).

${ }^{17}$ E. A. Dobisz, D. Kercher, M. Grobis, O. Hellwig, E. E. Marinero, D. Weller, and T. R. Albrecht, J. Vac. Sci. Technol. B 30, 06FH01 (2012).

${ }^{18}$ A. Stierle, T. Koll, and H. Zabel, Phys. Rev. B 58, 5062 (1998).

${ }^{19}$ J. Grabis, A. Nefedov, and H. Zabel, Rev. Sci. Instrum. 74, 4048 (2003).

${ }^{20}$ A. I. Figueroa, J. Bartolomé, L. M. García, F. Bartolomé, C. Magén, A. Ibarra, L. Ruiz, J. M. Gonzâlez-Calbet, F. Petroff, and C. Deranlot, J. Appl. Phys. 107, 09B508 (2010).

${ }^{21}$ K. Buschow, J. Appl. Phys. 54, 2578 (1983).

${ }^{22}$ Binary Alloy Phase Diagrams, 2nd ed., edited by T. B. Massalski, H. Okamoto, P. R. Subramanian, and L. Kacprzak (ASM International, 1990).
${ }^{23}$ M. M. Schwickert, G. Y. Guo, M. A. Tomaz, W. L. OBrien, and G. R. Harp, Phys. Rev. B 58, R4289 (1998).

${ }^{24}$ F. Brüssing, M. Ewerlin, A. Abrudan, and H. Zabel, Phys. Rev. B 88, 094431 (2013).

${ }^{25}$ A. Scherz, "Spin- dependent X-ray absorption spectroscopy of 3D transition metals: Systematics and application," Ph.D. thesis (Freie Universität Berlin, 2003).

${ }^{26}$ J. Stöhr and H.-Christoph Siegmann, Magnetism: From Fundamentals to Nanoscale Dynamics, Springer Series in Solid State Sciences (Heidelberg, 2007).

${ }^{27}$ P. Grünberg, J. Phys.: Condens. Matter 13, 7691 (2001).

${ }^{28}$ D. V. Talapin, E. V. Shevchenko, C. B. Murray, A. V. Titov, and P. Král, Nano Lett. 7, 1213 (2007)

${ }^{29}$ S. Mørup, M. Fougt Hansen, and C. Frandsen, Beilstein J. Nanotechnol. 1, $182(2010)$.

${ }^{30}$ T. R. McGuire and C. J. Kriesman, Phys. Rev. 85, 452 (1952). 